\title{
Influence of the shaper parameters on the characteristics of acceleration pulses reproduced by mechatronic shock machines
}

\author{
Denis Shtreker*, Mikhail Polishchuck, and Arkadii Popov \\ Peter the Great St. Petersburg Polytechnic University, Politechnicheskaya str., 29, 195251 St. Petersburg, Russia
}

\begin{abstract}
In this paper the influence of the shaper parameters on the characteristics of acceleration pulses (peak value and duration) reproduced on mechatronic shock machines is analyzed. A comparison of the acceleration pulses obtained experimentally and by computational methods is presented. Recommendations for clarifying the requirements for methodological calculations of pulse parameters are given. The dependence of the elastic force of the shock pulse shaper on its deformation is presented. The influence of this characteristic on the peak value and duration of the acceleration pulse is estimated. The influence of the height of the shaper on the parameters of the acceleration pulses is analyzed. The concept of a device that allows you to automatically change the height of the shaper to obtain a wider range of acceleration pulses is presented. The interaction of the shock table with the shaper by means of computer modeling is modelled. Conclusions about the dependences of the duration and peak value of the acceleration pulses on the parameters of the shaper (stiffness and height) are drawn. Recommendations for selecting the parameters of the shaper to obtain acceleration pulses with the desired parameters are given.
\end{abstract}

\section{Introduction}

Shock testing is a prerequisite for the commissioning of most objects surrounding a person. It is most expedient to carry out such tests in laboratory conditions on special mechatronic shock machines, with the help of which it is possible to reproduce the acceleration pulses with the necessary (set by the operator) parameters. In practice, the generation of pulses with the required parameters is difficult due to the inaccuracy of the models used to select the settings of such machines. In particular, analytical models usually do not consider the friction force in the guides of a mechatronic shock machine and the unstable rigidity of the hyper elastic shock pulse shaper.

In $[1,2]$, for modeling shock tests, the shaper is represented as a spring with linear stiffness. The approximate calculations based on this method are very different from the actual experimental data.

In [3], an empirical formula for selecting a shaper for reproducing pulses of the required parameters is presented. However, it is noted that the formula can only be used for the first iteration of the shaper selection, which will require further adjustment.

In [4], an approach is proposed for the selection of the shaper, which is based on the determination of its elastic force from the reproducible shock pulse. It allows you to estimate this force for a particular shaper from a single experiment. However, the problem is that the acceleration pulses, even in the same mode, are very different, and a large number of experiments are necessary to obtain an adequate characteristic.

There are also known works devoted to the description of a hyper elastic material using various nonlinear models $[5,6,7]$. In the article [8], the authors used the Arruda-Boyce model to describe the behavior of the hyper elastic material of the and simulated shock tests using the finite element method. The main disadvantages of this method are high computational costs and certain predictable errors.

A similar model, but using the two-parameter Mooney-Rivlin model, is presented in $[9,10,11]$. They allow us to estimate the parameters of shock pulses with higher accuracy, but even in this case, the error can be from $15 \%$ to $40 \%$.

In this paper, the influence of various parameters of the shaper on the characteristics of acceleration pulses reproduced by mechatronic shock machines is analyzed. The possibility of regulating the characteristics of the acceleration pulses by changing the parameters of the shaper is estimated.

\section{Influence of the shaper material on the parameters of the acceleration pulses}

\subsection{Methods of conducting experiments}

* Corresponding author: shtreker.denis@gmail.com 
The preliminary estimation of the parameters of the acceleration pulses is an urgent task today. This estimation allows you to optimize the time spent, reduce the number of iterations for reproducing the required (operator-defined) shock pulse, and obtain the desired pulse characteristics with greater accuracy. To assess the influence of the shaper parameters on the characteristics of the acceleration pulses, experiments were carried out on the Lansmont M95/115 mechatronic shock machine (Figure 1).

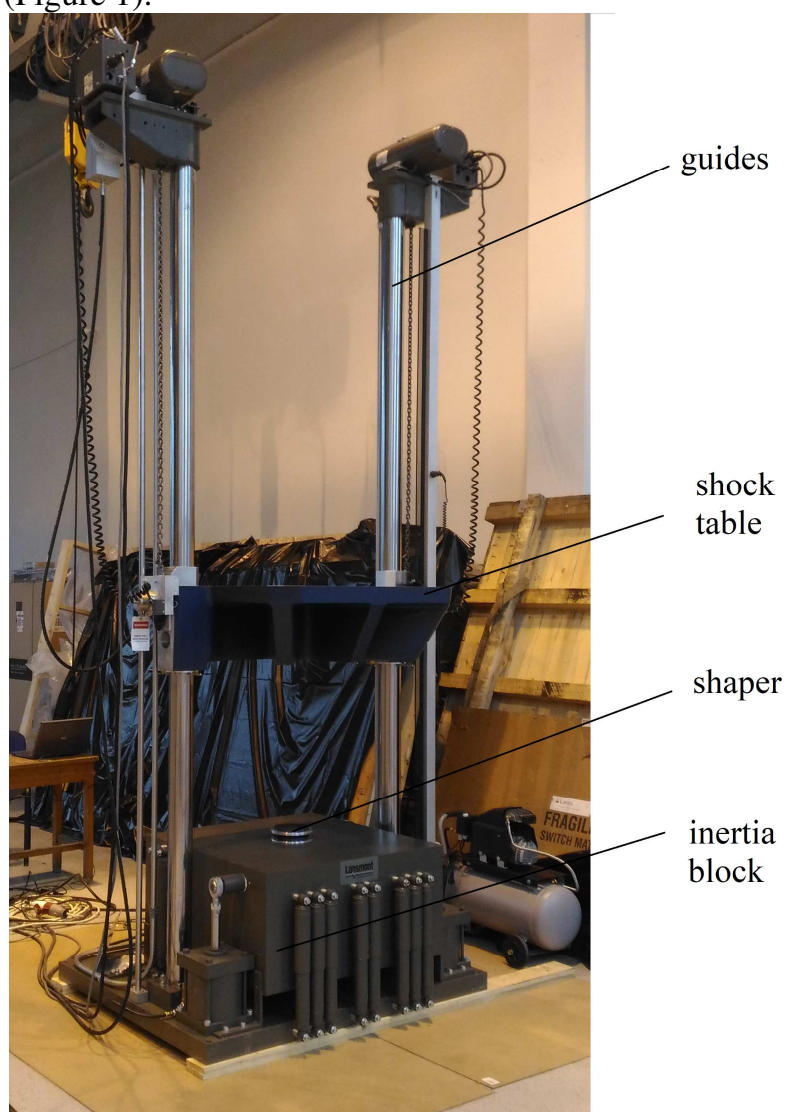

Fig. 1. Lansmont M95/115 mechatronic shock machine

The main elements of the mechatronic shock machine are the inertia block, the shock table and the guides along which the table moves directly.

To implement the process of conducting shock tests, it is necessary to implement the following algorithm. The object under study is fixed on the upper surface of the shock table. A shock pulse generator is installed on the upper surface of the inertia block. The pulse is created by the shock interaction of the lower surface of the table with the shaper. After reproducing the shock pulse, you can raise the shock table to the height required for a new experiment, or finish working with the machine.

\subsection{Nonlinear dependence of the elastic force on the deformation of the shaper}

The discrepancy between the data obtained by computational methods and the experimental data is a big problem when selecting shapers for reproducing pulses with the required parameters. Figure 2 shows the pulses obtained using the techniques $[1,2]$ and the real experimental pulses obtained on the Lansmont M95/115 mechatronic shock machine.

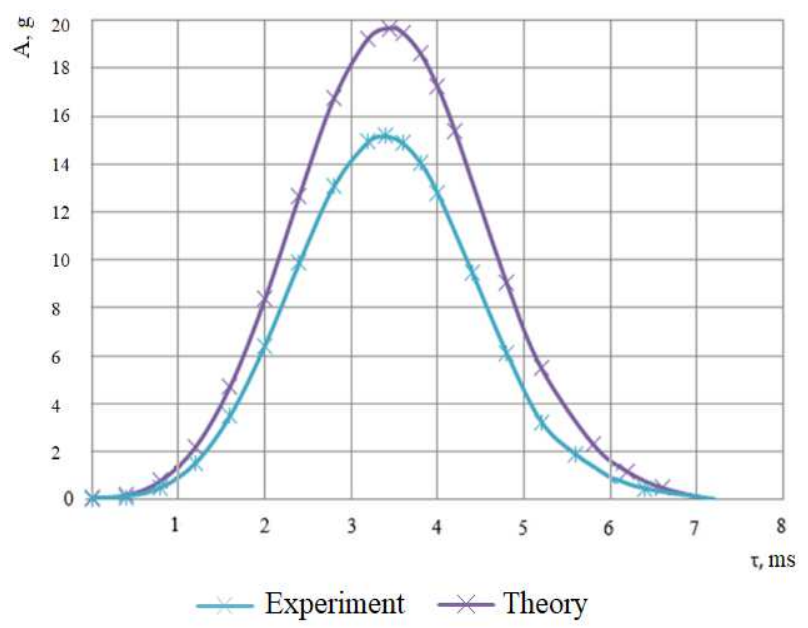

Fig. 2. Comparison of experimental and theoretical parameters of acceleration pulses

With a given set of parameters characteristic of one of the modes of the Lansmont machine (table weight $431 \mathrm{~kg}$; table drop height $-0.05 \mathrm{~m}$; dynamic force rating of the shaper - $45300 \mathrm{kgf}$; shaper height - $25 \mathrm{~mm}$; shaper diameter $-152 \mathrm{~mm}$ ), the peak pulse values differ by more than $20 \%$. Such differences can be explained by the inaccuracy of the description of the forces that arise in the «shock table-shaper» system at the moment of shock (Figure 3).

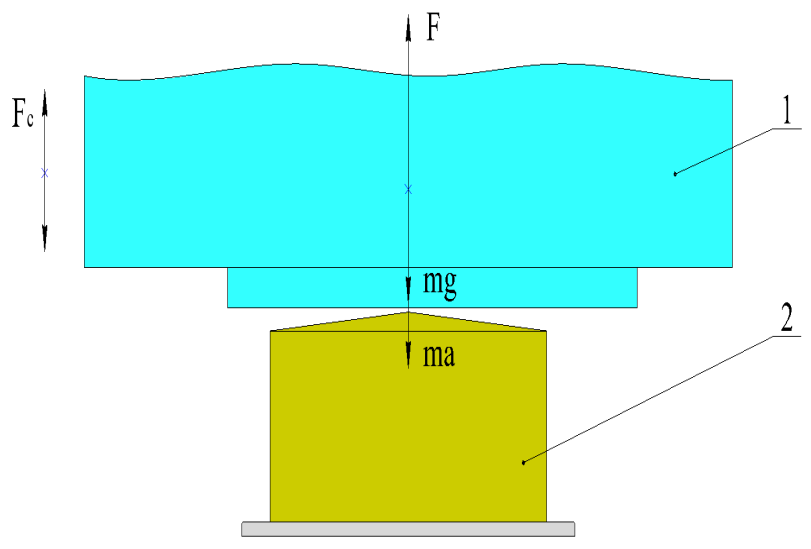

Fig. 3. Diagram of forces in the «shock table-shaper» system at the moment of shock: 1 - shock table; 2 - shock pulse shaper

The scheme shown in Figure 3 corresponds to the equation:

$m a=F-m g \pm F_{c}$

where $m$ is the mass of the shock table, $a$ is the shock acceleration, $F$ is the elastic force of the shaper, $g$ is the acceleration of gravity $\left(g=9.81 \mathrm{~m} / \mathrm{s}^{2}\right), F_{c}$ is the friction force in the guides.

In practice, the stiffness of a hyper elastic shaper is not a constant. The elastic force depends on the 
deformation of the shaper non-linearly (Figure 4), which significantly affects the results of the experiment [12, 13].

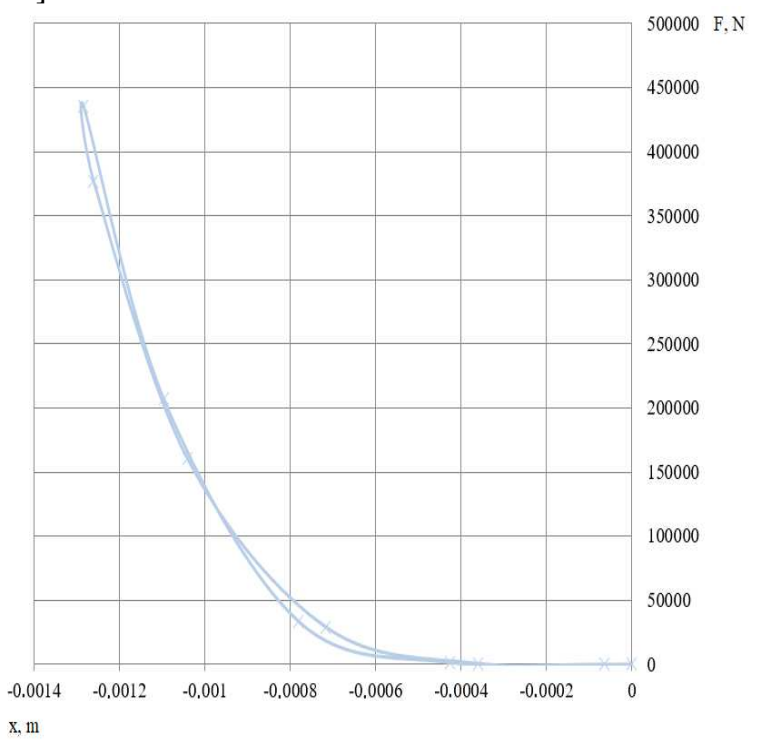

Fig. 4. Dependence of the elastic force $F$ on the deformation of the shaper $x$

On the basis of the conducted experiments, the dependence of the elastic force on the deformation for the studied shaper was determined (the diameter of the shaper is $152 \mathrm{~mm}$; the shaper height is $25 \mathrm{~mm}$; the dynamic force rating of the shaper is $45300 \mathrm{kgf}$ ):

$F=2 \cdot 10^{17} \cdot x^{4}-2 \cdot 10^{12} \cdot x^{3}-7 \cdot 10^{10} \cdot x^{2}-9 \cdot 10^{6} \cdot x+3138$.

The hysteresis of this characteristic can be explained by the internal friction in the material of the shaper and the friction in the guides during shock and rebound. Based on the model presented in [14], the obtained dependence can be used to refine the output parameters of the experiment. Thus, the simulated shock pulses, considering the nonlinearity of the elastic force characteristic, are shown in Figure 5.

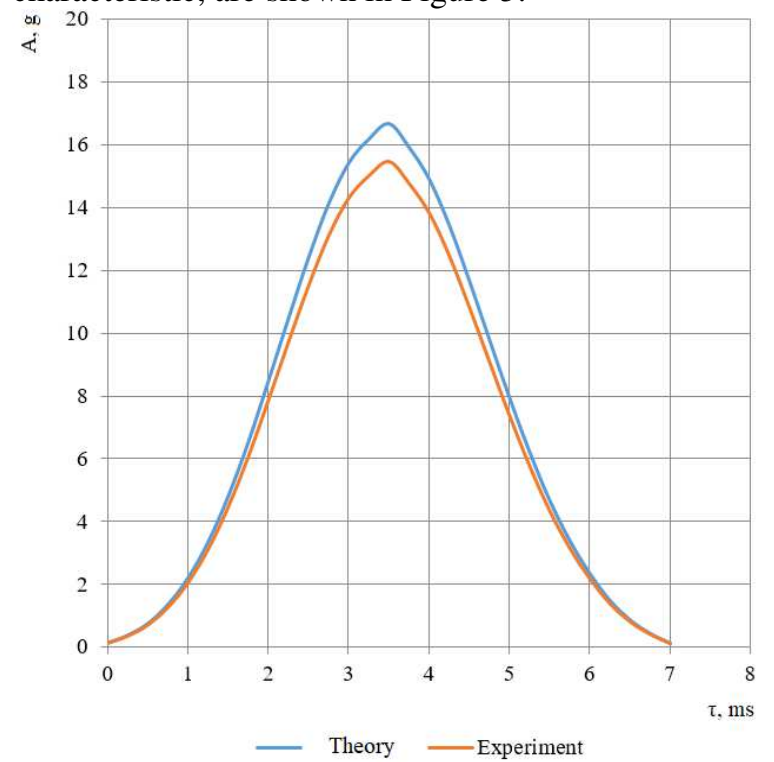

Fig. 5. Comparison of experimental and theoretical parameters of acceleration pulses considering the non-constant stiffness of the shaper
The use of the nonlinear characteristic of the elastic force of the shaper reduces the difference between the theoretical estimates and the experimental data to $10 \%$, which can be seen from Figure 5.

\section{Influence of the shaper height on the parameters of the acceleration pulses}

\subsection{Device for adjusting the height of the shaper}

Adjusting the parameters of the elastic force of the shaper opens up wide possibilities for varying the parameters of the acceleration pulses. Changing this characteristic is possible by changing the geometric dimensions or the material of the shaper. Also, you can change the characteristic by limiting the deformation of the side surface of the shaper. A similar principle is implemented in SHINYEI PDST-Series percussion machines [15], where the shaper is limited by means of a special device, the height of which is manually adjusted by the operator.

Employees of the Higher School of Automation and Robotics of the Peter the Great St. Petersburg Polytechnic University proposed the concept of a device that allows automating this process. The device is shown in Figure 6.

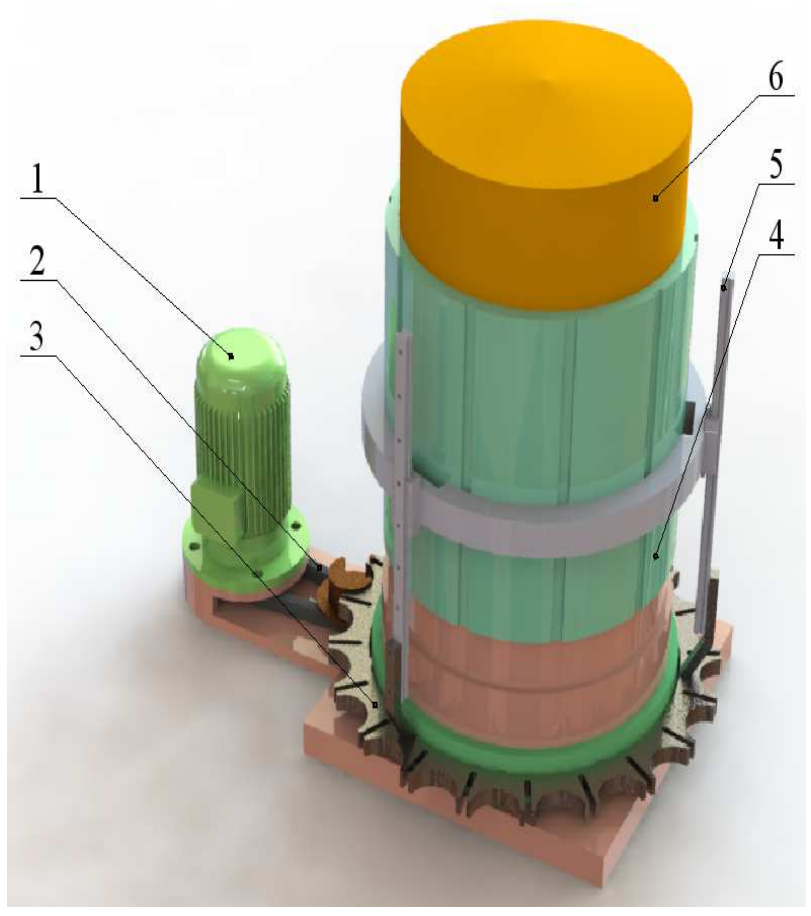

Fig. 6. Device for regulating the lateral deformation of shock pulse shapers

The device is driven by an electric motor 1 through a belt drive 2 and a subsequent Maltese mechanism 3 . The Maltese mechanism provides rotation and fixation of the drum 5. The Maltese wheel is connected to the drum by means of vertical linear guides 4 . The vertical movement 
of the drum 5 occurs by converting the rotational movement of the Maltese mechanism into a translational one by means of a «screw-nut» transmission. The nut is the drum itself, and the screw is the support of the shaper 6. In fact, limiting the deformation is equivalent to changing the working height of the shaper. Therefore, the use of such a device allows you to automatically adjust the height of the shaper, which is especially important when working with mechatronic impact machines of multiple action.

\subsection{Investigation of the parameters of the shaper by means of computer modeling}

The deformation process of the shaper is modeled using the ANSYS Workbench tools. For correct modeling, it is necessary to experimentally determine the dependence of the static characteristic of the deformation of the shaper material on the load applied during its compression. A similar characteristic was obtained in the laboratories of Peter the Great St. Petersburg Polytechnic University for a sample of a simple cylindrical former material. The dependence obtained experimentally is shown in Figure 7.

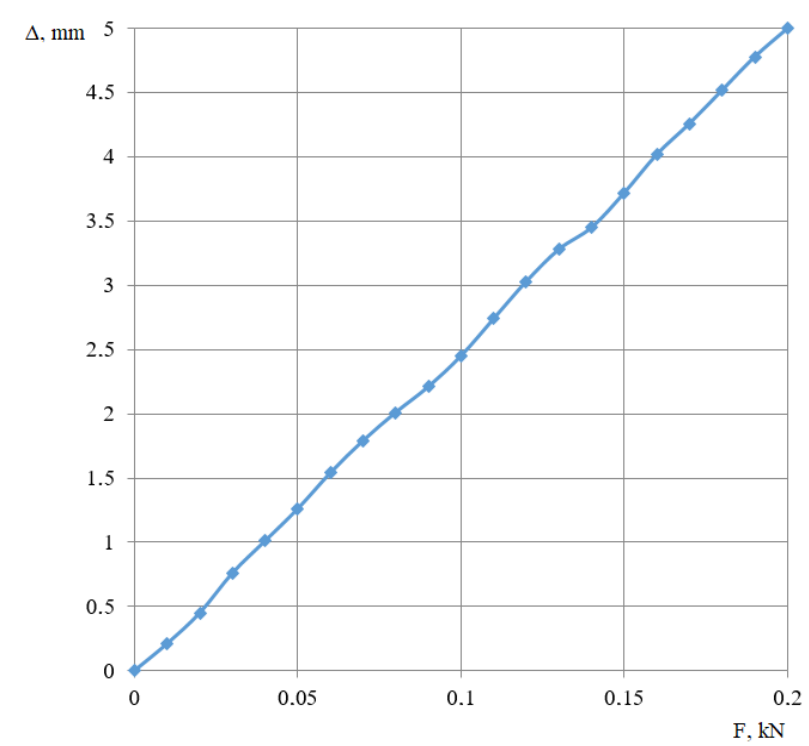

Fig. 7. The dependence of the static characteristic of the deformation of the shaper material $\Delta$ on the load applied during its compression $\mathrm{F}$

The experimental characteristic can be used as the initial information for selecting the parameters of the material model in the ANSYS. A fairly good match between the computer simulation and the experiment was obtained for the five-parameter Mooney-Rivlin model. A computer model of the interaction of the impact table and the shaper is constructed for a shaper with a diameter of $152 \mathrm{~mm}$, considering the possibility of adjusting the «working» height of the shaper. Figures 8 and 9 show the deformations of the shaper for two cases, when the «working» height of the shaper is 25 $\mathrm{mm}$ and $76 \mathrm{~mm}$.

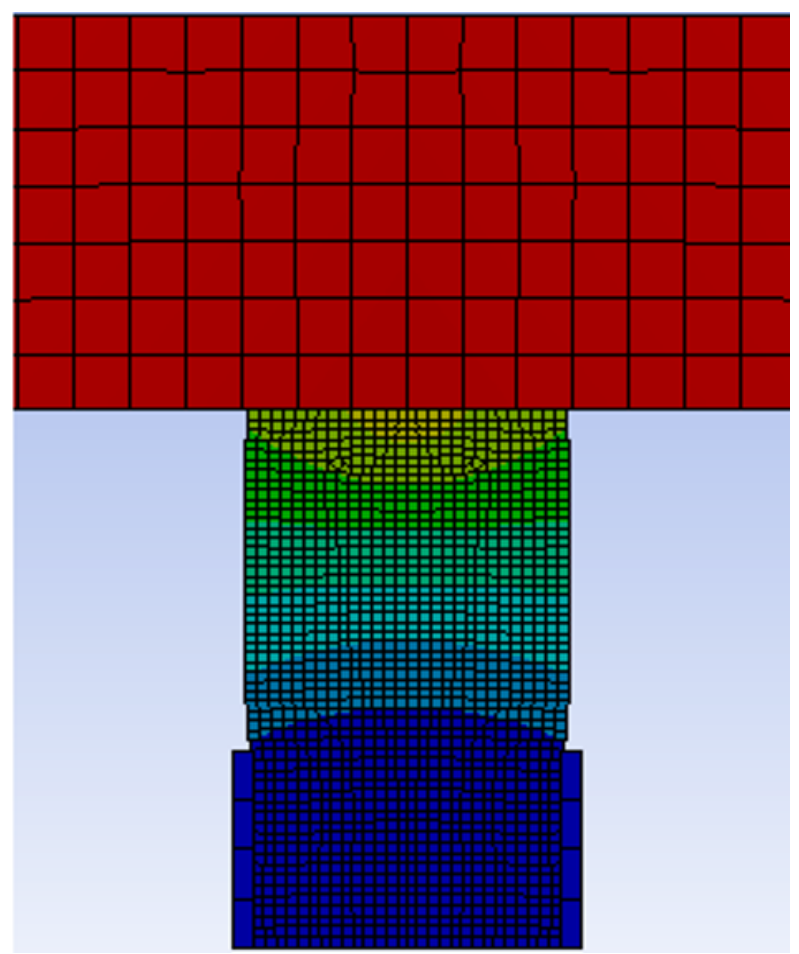

Fig. 8. Deformed state of the shaper at the «working» height of $76 \mathrm{~mm}$

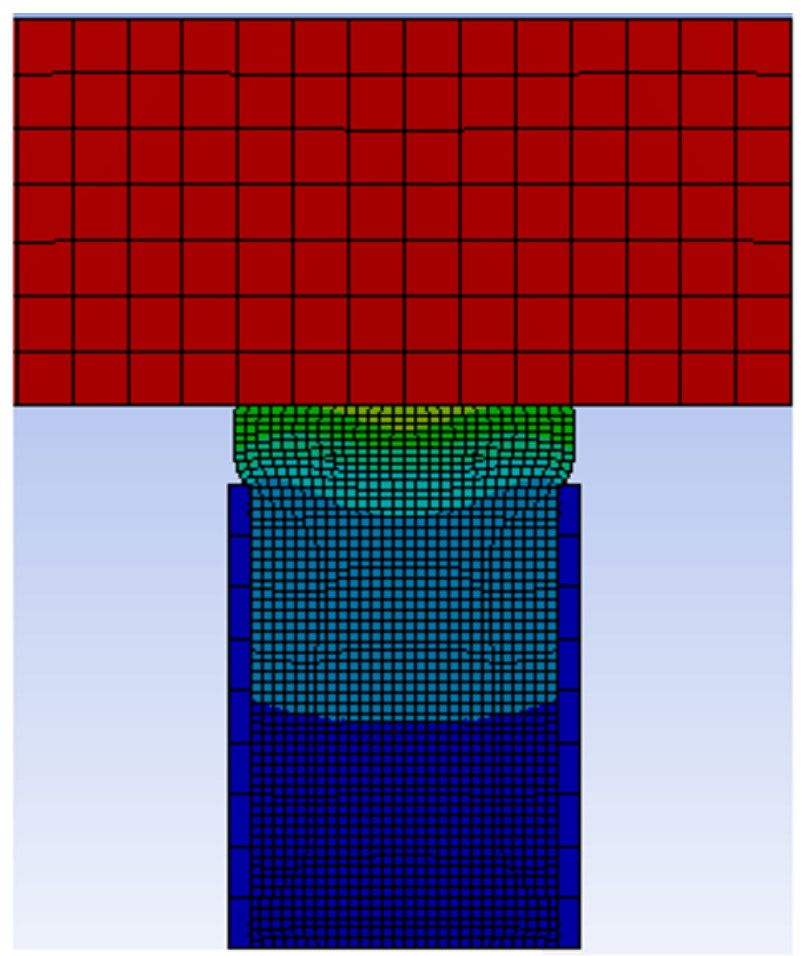

Fig. 9. Deformed state of the shaper at the «working» height of $25 \mathrm{~mm}$

From the simulation results, it is possible to notice differences in the degree of deformed states of the shaper at different heights of the limiting device. This difference is reflected in the dependence of the elastic strength of the shaper material on the deformation. Graphs of such dependencies are shown in Figure 10. 


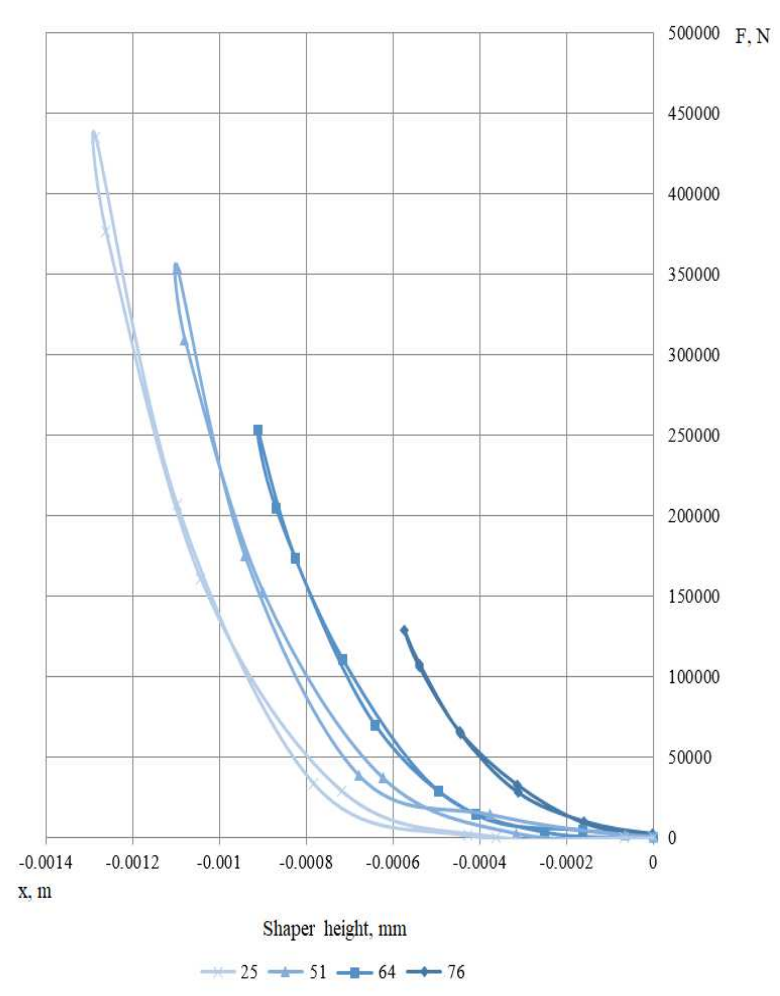

Fig. 10. Dependence of the elastic force $F$ on the deformation of the shaper $x$

The influence of the limiting device on the dependence of the elastic force of the shaper is presented in Table 1.

Table 1. Dependence of the elastic force $F$ on the deformation of the shaper $x$

\begin{tabular}{|c|c|}
\hline $\begin{array}{l}\text { Shaper } \\
\text { height, } \\
\text { mm }\end{array}$ & $\begin{array}{l}\text { Dependence of the elastic force } F \text { on the } \\
\text { deformation of the shaper } x\end{array}$ \\
\hline 25 & $\begin{array}{c}F(x)=7 \cdot 10^{17} \cdot x^{4}+6 \cdot 10^{13} \cdot x^{3}+10^{11} \cdot x^{2}+3 \cdot 10^{7} \cdot x+ \\
951\end{array}$ \\
\hline 51 & $\begin{array}{c}F(x)=2 \cdot 10^{17} \cdot x^{4}-10^{14} \cdot x^{3}+5 \cdot 10^{9} \cdot x^{2}+10^{6} \cdot x+ \\
898\end{array}$ \\
\hline 64 & $\begin{array}{c}F(x)=3 \cdot 10^{17} \cdot x^{4}+10^{12} \cdot x^{3}-6 \cdot 10^{10} \cdot x^{2}-2 \cdot 10^{7} \cdot x+ \\
3684\end{array}$ \\
\hline 76 & $\begin{array}{c}F(x)=2 \cdot 10^{17} \cdot x^{4}-2 \cdot 10^{12} \cdot x^{3}-7 \cdot 10^{10} \cdot x^{2}-9 \cdot 10^{6} \cdot x+ \\
3138\end{array}$ \\
\hline
\end{tabular}

This dependence allows you to change the amplitude and duration of the shock pulses by adjusting the height of the device.

To illustrate this approach, the results of modeling in the Matlab Simulink for shapers made of the same material that differ only in the «working» height of the shaper are presented. Figure 11 shows the acceleration pulse at the «working» height of the shaper of $25 \mathrm{~mm}$.

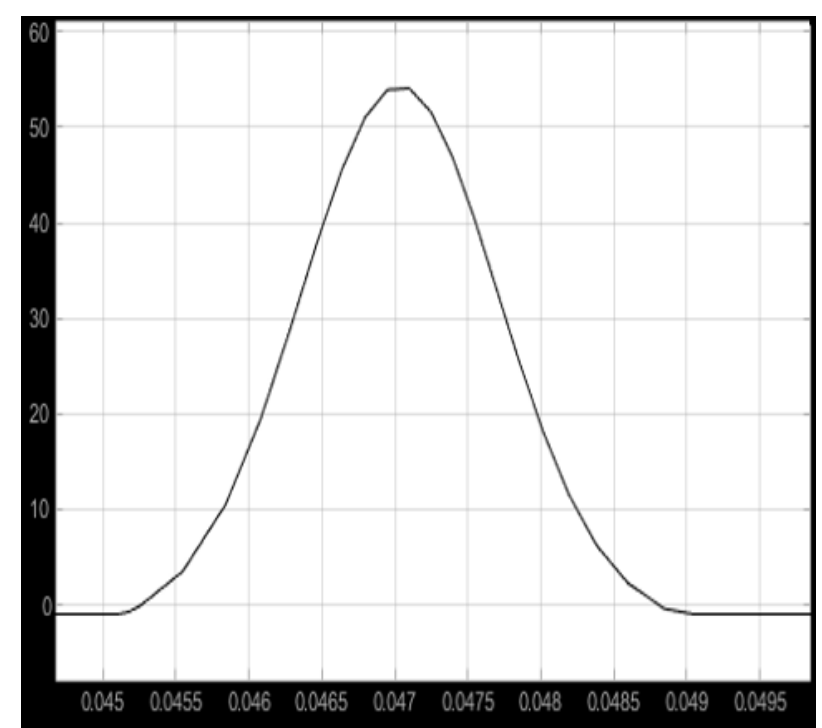

Fig. 11. Acceleration pulse at the «working» height of the shaper $25 \mathrm{~mm}$

The result of the simulation at the «working» height of the shaper $51 \mathrm{~mm}$ is shown in Figure 12.

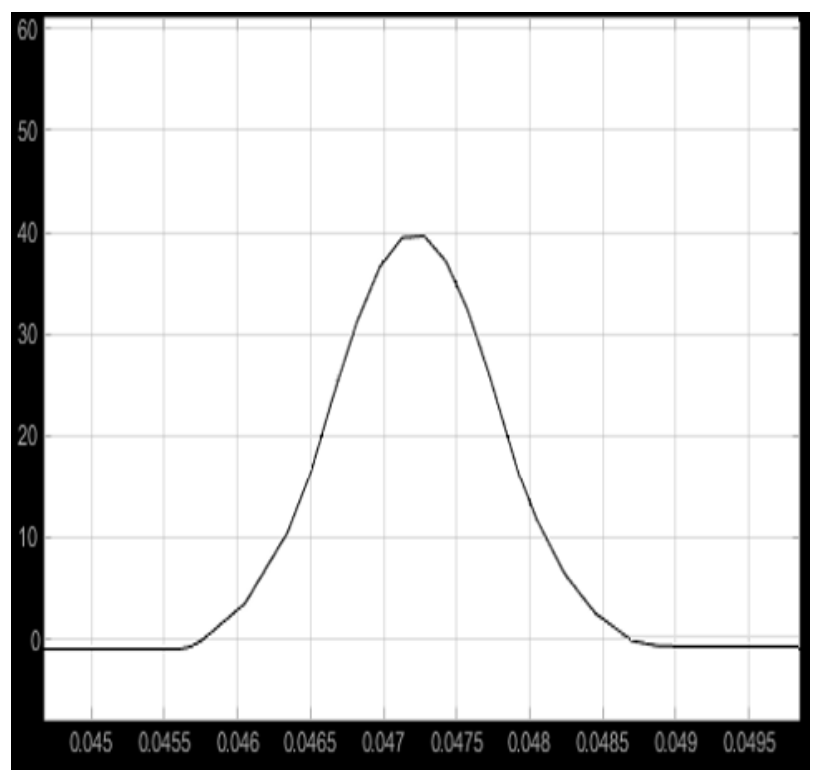

Fig. 12. Acceleration pulse at the «working» height of the shaper $51 \mathrm{~mm}$

The simulation results for various parameters of the shaper height are presented in Table 2.

Table 2. Parameters of acceleration pulses for different heights of the shaper

\begin{tabular}{|c|c|c|}
\hline $\begin{array}{c}\text { Shaper height, } \\
\mathrm{mm}\end{array}$ & Peak value, g & Duration, ms \\
\hline 25 & 57 & 3.9 \\
\hline 51 & 40 & 4.4 \\
\hline 64 & 25 & 5.6 \\
\hline 76 & 19 & 6.7 \\
\hline
\end{tabular}

Based on the simulation results, it can be seen that changing the height of the shaper allows you to change the parameters of the acceleration pulses several times. 


\section{Conclusion}

When estimating the parameters of the acceleration pulses, it is necessary to consider the characteristics of the shaper. The variable rigidity of the shaper complicates analytical calculations, but allows you to adjust the parameters of the required acceleration pulses in wide ranges solely due to the characteristics of the shaper. Changing the parameters is possible both by changing the material of the hyper elastic shaper and by changing its "working" height. The use of a device to limit the lateral deformation of the shaper allows you to change the amplitude and duration of the shock pulses in a wide range. So, at the working height of the shaper of $25 \mathrm{~mm}$, the peak value of the acceleration pulse is $57 \mathrm{~g}$. When the height of the shaper increases to $76 \mathrm{~mm}$, the amplitude decreases 3 times and is $19 \mathrm{~g}$. The pulse duration, on the contrary, increases with increasing the height of the shaper $(6.7 \mathrm{~ms}$ at a height of $76 \mathrm{~mm}$ versus $4.2 \mathrm{~ms}$ at a height of $25 \mathrm{~mm})$.

\section{References}

[1] C. Lalanne Mechanical Vibration and Shock Analysis, 2 (2009)

[2] H. Chen, M.M. Mu, X.H. Wang, D.Y. Wang, Environ Technol, 52 (2013)

[3] L.K. Stewart, B. Durant, J. Wolfson, G.A. Hegemier, International Journal of Impact Engineering, 69 (2014)

[4] J. Lü, X.H. Yue, H.J. Huang, China Rubber Indust, 55(1) (2008)

[5] J.Q. Qin, Y.L. Yang, C.C. Di, S.Q. Guan, Trans Tech Publications, 117 (2012)

[6] M.C. Boyce, Rubber Chem Technol, 69 (5) (1996)

[7] Y.J. Mao, Environ Technol, 2002 (5) (2002)

[8] Y.L. Yang, J.Q. Qin, C.C. Di, T.K. Cui, J Vibr Shock, 33 (2) (2014)

[9] J. Wen, Ch. Liu, H. Yao, B. Wu, International Journal of Impact Engineering, 120 (2018)

[10] H. Li, W. Chen, H. Hao, International Journal of Impact Engineering, 145 (2020)

[11] G. Wang, Y. Xiong, W. Tang, International Journal of Mechanical Sciences, 77 (2013)

[12] A. Zhang, , Proc. ECTC (2014)

[13] D.S Shtreker, A.N Popov, D.V Reshetov, G.A Utkin J. Phys.: Conf. Ser. 1753 (2021)

[14] A.N. Popov, M.N. Polishchuck, A.K. Vasiliev, International Review of Mechanical Engineering, 14 (2) 2020

[15] Technical specifications of the SHINYEI PDST Series Shock Tower test stand Retrieved from: https://shinyei-tm.com/product_ST_PDST.html 